\title{
Ultrashort Pulse Generation from an Integrated Single-Chip Dye Laser
}

\author{
Mitsuo Maeda, Yuji Oki, and Katsumi Imamura
}

\begin{abstract}
In distributed-feedback (DFB) dye lasers pumped by a nanosecond-pulse laser, a train of picosecond spikes is generated by the relaxation oscillation. A single spike with a pulse duration of $50 \mathrm{ps}$ is extracted in a plastic-waveguide DFB dye laser using a quenching technique. To compress this to femtosecond pulse, a new type of integrated pulse compressor is proposed, and its performances are analyzed. The compressor is composed of a plastic waveguide in which amplifier dye and saturable dye are alternatively doped. Computer simulation shows that the 50-ps pulse is compressed to 120 fs by passing through four stages of amplifier and saturable absorber pair. Using this scheme, we expect to construct a very compact and simple ultrashort pulse generator in which the whole system is integrated on a chip of a plastic waveguide.
\end{abstract}

\section{INTRODUCTION}

D YE LASERS have greatly contributed to the progress of the femtosecond (fs) technology. The stability in the fs pulse generation is much improved by the use of new solid-state tunable devices, such as the Ti:sapphire laser, the Cr:LiSAF laser and optical parametric oscillators. In this case, the short pulse generation is based on the mode-locking technique. The mode-locking technique essentially requires CW pumping and a long cavity to grow up short pulses, so that the system tends to be large and complicated.

On the other hand, researchers in the Max-Planck-Institute in Gottingen, Germany, have reported many papers on the short pulse generation with pulsed dye lasers based on the transient pulse compression technique [1], [12]. In shortcavity or distributed-feedback (DFB) dye lasers pumped by a laser with nanosecond (ns) pulse duration, spiking caused by the relaxation oscillation is observed. Each spike has a pulse duration of several tens of picoseconds (ps) typically. Bor et al. demonstrated the single spike extraction by means of the quenching technique, injecting an optically delayed pulse generated by another dye laser [6], [8]. Furthermore, Szatmári et al. compressed these ps pulses to $100 \mathrm{fs}$ through the pumping of another achromatic DFB dye laser [10]-[12].

The pumping source of this type of ultrashort pulse generator is the ns-pulse lasers, such as Nd:YAG, excimer, and $\mathrm{N}_{2}$ lasers, and essentially the short cavity of the dye laser is important to generate short pulses in contrast to the mode-locking technique. However, because the procedure of the single-pulse extraction and the pulse compression is not so simple, the

Manuscript received December 10, 1996; revised August 4, 1997.

The authors are with the Department of Electronic Device Engineering, Graduate School of Information Science and Electrical Engineering, Kyushu University, Fukuoka 812-81, Japan.

Publisher Item Identifier S 0018-9197(97)08413-3. system becomes large and complicated as in the mode-locking case. This type of system is also commercialized, but not so popular as the mode-locking type now.

In this paper, we developed a DFB dye laser with a plastic waveguide structure. This laser generated periodical ps spikes by the pumping of a $Q$-switched Nd:YAG laser. For the extraction of the first spike, we used a novel quenching scheme and obtained a single 50-ps FWHM pulse. To compress this to sub-ps pulse, a new type of integrated pulse compressor was proposed and estimated the performances of this device with the photon transport equation.

The proposed ultrashort pulse generator is a very small and simple device, and a whole system can be integrated on the chip of a plastic waveguide. The proposed scheme can also be applied to solid-state tunable lasers with a short relaxation time, such as color center lasers and a Ce:LiSAF laser.

\section{EXtraction of A Single Spike In DFB Dye LASER}

In 1971, Kogelnik and Shank first demonstrated the DFB dye laser by the use of a dye-doped gelatin film with a grating inside [13]. Shank et al. showed that the DFB laser action was also possible by making periodic fringes of the pump beam inside the dye cell [14]. Changing the incidence angle between interfering pump beam, the dye laser generated tunable narrow-band emission.

The output pulses of these DFB dye lasers have a structure of periodic pulse train [14], [1], [2]. These spikes are caused by the relaxation oscillation which is a well-known phenomena in pulsed solid-state lasers. Analysis of the rate equation shows that a series of periodical spikes with a duration of 10-100 ps can be generated in lasers with a short fluorescence lifetime $(\tau \sim \mathrm{ns})$ and a short cavity lifetime $\tau_{c}$. Although the DFB structure is not essential to generate these ps spikes, the short active length of the DFB laser is suitable to shorten the effective $\tau_{c}$ value.

The DFB dye laser developed in our laboratory was a waveguide type in which a poly-methylmethacrylate (PMMA) film doped Rhodamine $6 \mathrm{G}$ dye was coated on a glass substrate. The film was spin-coated at the prepolymerized stage, then fully polymerized by the heating. The thickness of the film was in the range of $1.6-3 \mu \mathrm{m}$. The dye concentration was estimated from the measurement of the absorbance of the dye, and the concentration range was from 0.5 to $5 \times 10^{-2} \mathrm{~mol} / \mathrm{l}$.

The experimental setup of DFB laser is shown in Fig. 1(a). The pump source was a $Q$-switched Nd:YAG laser (Spectra Physics GCR-12). The frequency-doubled pulse (532 nm, $6 \mathrm{~ns}$ FWHM, $10 \mathrm{~Hz}$ ) was shaped into a sheet beam with a spot size 


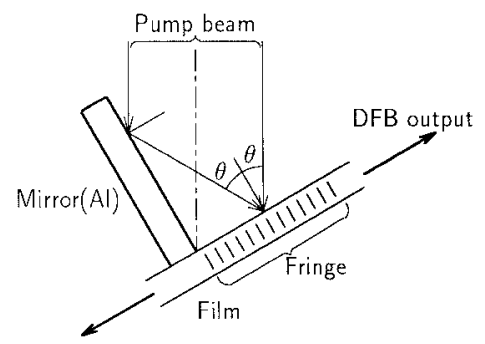

(a)

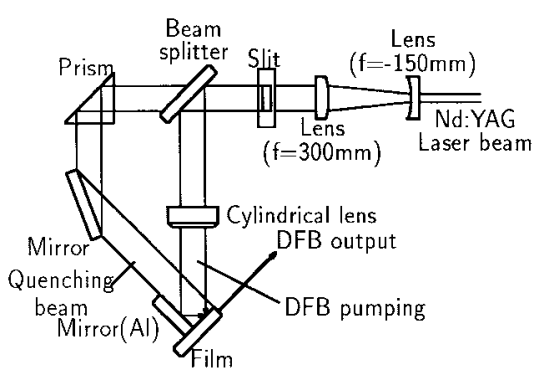

(b)

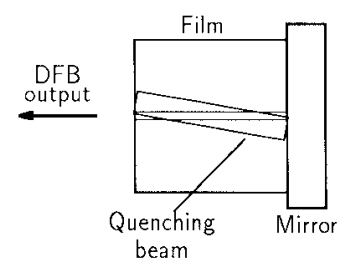

(c)

Fig. 1. (a) Pumping scheme of thin-film DFB dye laser, (b) experimental setup for single-spike extraction, and (c) tilted quenching beam on chip.

of $0.5 \times 10 \mathrm{~mm}^{2}$ on the film. A half of the beam was reflected by the mirror vertically placed on the film, and the interference fringes were formed inside the film, and DFB laser action was attained.

The wavelength of the DFB laser at an incidence angle of $\theta$ is given by

$$
\lambda=\frac{n \lambda_{p}}{m \sin \theta}
$$

where $\lambda_{p}$ is the pump wavelength, $n$ is the refractive index of the waveguide, and $m$ is the order of diffraction. The emission spectra of the DFB dye laser are shown in Fig. 2 as a function of the incident angle of $\theta$ for $m=2$. The spectral bandwidth measured by a Fabry-Perot interferometer was 70-100 pm.

The typical output energy was $20 \mu \mathrm{J} /$ side for the pump energy of $300 \mu \mathrm{J}$. The threshold energy was less than $100 \mu \mathrm{J}$. The dye degradation was not so severe over $5 \times 10^{4}$ shot operation, but after $10^{4}$ shot operation at a fixed angle of $\theta$, DFB laser action was obtained without interference fringes, even in shading one of the beams. In this case, a permanent grating was printed inside the waveguide probably by the thermal effect.

The output pulse shape of the DFB dye laser was monitored by a ps streak camera (Hamamatu C3194). A typical trace is shown in Fig. 3(a). These spikes are always observed, but the pulse height is not regular. One of the reasons for this irregularity is that the pulse shape of the $\mathrm{Nd}$ :YAG laser is not

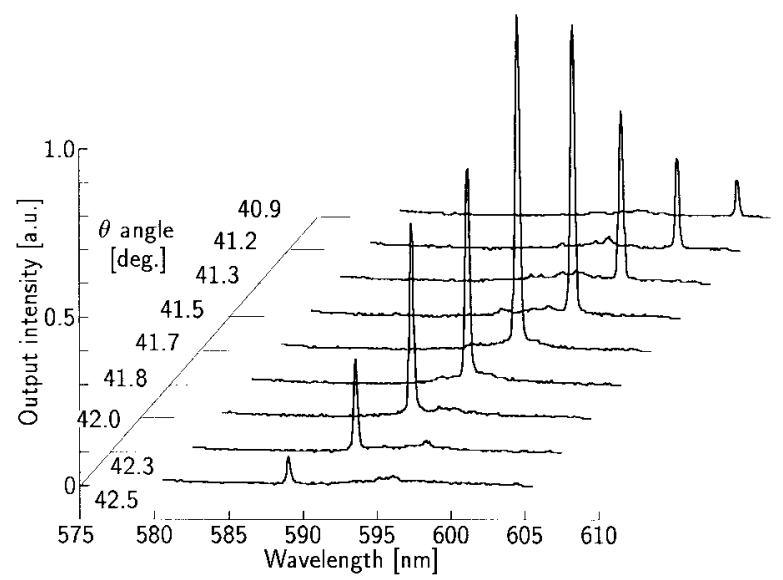

Fig. 2. Output spectra of DFB dye laser as a function of incidence angle $\theta$.

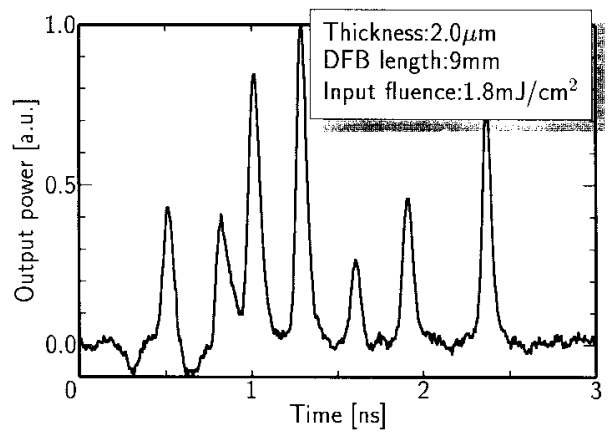

(a)

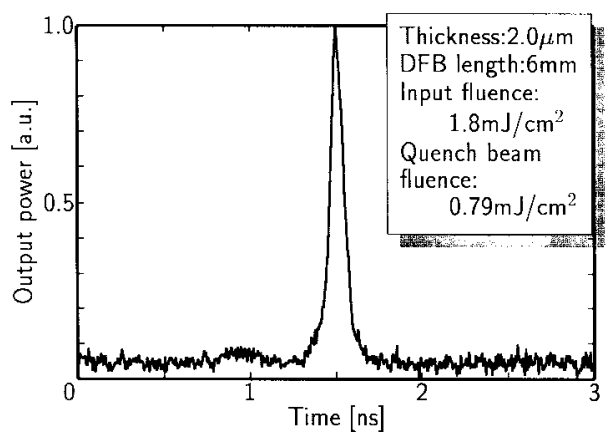

(b)

Fig. 3. (a) Typical streak camera traces of spiking and (b) extracted single spike.

smooth. If an injection-seeded Nd:YAG laser with a smooth output pulse is used for pumping, this may be improved.

The duration of each spike and the period between spikes are decreased by reducing the active length of the DFB laser from 10 to $4 \mathrm{~mm}$. They also decreased by increasing the pump energy. Although these were consistent with the rate equation analysis [1], [2], quantitative comparison was difficult because of the irregularity in the pump pulse shape.

The single spike can be generated by decreasing the pump power to near threshold. In that case, however, the pulse duration increases, the pulse energy decreases, and the stability tends to be poor. Bor et al. tried the first spike extraction by quenching successive spikes with the injection of a delayed optical pulse [6], [9]. They used another dye laser beam for 
the quenching, and the population inversion was quenched by taking out the energy to a tilted direction.

In the present experiment, we tried a simpler quenching technique, as shown in Fig. 1(b). The optically delayed quenching beam of the $\mathrm{Nd}$ :YAG laser was injected into the active region of the DFB laser from the vertical direction. In this case, the sheeted quenching beam is slightly tilted as shown in Fig. 1(c). The quenching beam destroys the grating formed in the waveguide, and the DFB laser action is stopped.

A single spike was successfully extracted, as shown in Fig. 3(b). The fluence of the quenching pulse was approximately $1 \mathrm{~mJ} / \mathrm{cm}^{2}$. The shortest pulse duration obtained in this experiment was 47 ps FWHM. Thus, the 6-ns pump pulse was compressed to $47 \mathrm{ps}$ with an integrated waveguide dye laser.

\section{INTEGRATED PULSE COMPRESSOR}

In order to obtain the fs pulses, the researchers of MaxPlanck-Institute have tried the traveling wave excitation [5], [7] and achromatic DFB laser pumped by a ps dye laser [10]-[12]. We propose a more simple and effective method suitable for the integrated dye laser. A very compact singlechip ultrashort-pulse generator can be constructed using the proposed method.

Passing a short laser pulse through a chain of saturable absorbers (SA's) and dye laser amplifiers alternatively, the pulse duration is gradually shortened [15], [16]. Taira and Yajima obtained a 0.8 -ps pulse by the compression of a 18-ps pulse through a two-stage SA-amplifier chain of DODCI (3, $3^{\prime}$-Diethyloxadicarbocyanine Iodide) and Rhodamine 6G [15]. In this case, the leading edge of the pulse is sharpened by SA, and the tail of the pulse is cut by saturated amplification. This is the same pulse-sharpening mechanism as observed during the build-up time of the passive mode locking in dye lasers [17].

However, the configuration of a multistage SA-amplifier chain is not so simple. This problem is solved by the technique of integration, as shown in Fig. 4(a). In Fig. 4(a), the SA (DODCI) and the laser dye (Rhodamine 6G) are alternatively doped in a PMMA waveguide, and the pump beam is shaded for the saturable absorbers. The whole system, including the single pulse generator described in the previous section, can be integrated in a single chip, as shown in Fig. 4(b), very compactly.

Next, we estimate the performances of the proposed pulse compressor shown in Fig. 4(a), applying photon transport equations to the SA and amplifier [17].

The following equations are assumed to describe the dye laser amplifier in a four-level system:

$$
\begin{aligned}
\frac{\partial p}{\partial t}+c \frac{\partial p}{\partial x} & =c p(\sigma q-\alpha) \\
\frac{\partial q}{\partial t} & =-c \sigma q p-\frac{q}{\tau}
\end{aligned}
$$

and the SA in a two-level system:

$$
\begin{aligned}
\frac{\partial p}{\partial t}+c \frac{\partial p}{\partial x} & =c p\left(\sigma_{a} n-\alpha_{a}\right) \\
\frac{\partial n}{\partial t} & =-2 c \sigma_{a} n p-\frac{n_{O}+n}{\tau_{a}}
\end{aligned}
$$

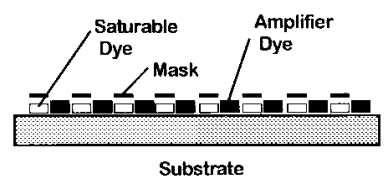

(a)

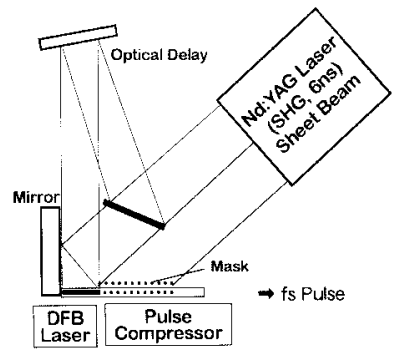

(b)

Fig. 4. (a) Structure of amplifier-SA chain integrated in a waveguide and (b) proposal of a single-chip ultrashort pulse generator.

where $p$ is the photon density, $q$ is the upper-state population in laser dye, $n$ is the population inversion in SA, $\sigma$ is the stimulated emission cross section of the laser dye, $\sigma_{a}$ is the absorption cross section of SA, $\alpha$ and $\alpha_{a}$ are the absorption coefficients of the laser dye and SA, $\tau$ is the fluorescence lifetime of the laser dye, $\tau_{a}$ is the relaxation time of SA, $n_{O}$ is the molecular density of SA, and $c$ is the light velocity. The pumping term of the amplifier is neglected, because the pulse duration is short enough.

The effect of the pumping in the amplifier is given by the initial condition of the upper-state population $q_{0}$ as follows. Before the amplifing pulse comes, the populatin $q_{0}$ is in the steady state with a balance between the pumping rate $W$ and the relaxation time $\tau$, that is,

$$
q_{0}=W \tau
$$

In the case of the side pumping, the pumping rate $W$ is given by

$$
W=\frac{1-\exp \left(-\sigma_{p} N d\right)}{h \nu V} P
$$

where $P$ is the pumping power, $\sigma_{p}$ is the absorption cross section of the laser dye at the pumping wavelength $\nu, N$ is the molecular density of the laser dye, $d$ is the thickness of the waveguide, $V$ is the active volume of the amplifier, and $h$ is the Planck's constant.

In the numerical calculation, the input pulse shape is assumed to be a Gaussian type with 50 ps FWHM. X-stage $\mathrm{SA}$-amplifer chain with a length of $1.5 \mathrm{~mm}$ (for each SA and amplifier) is assumed. The cross section of the waveguide is $3 \mu \mathrm{m} \times 500 \mu \mathrm{m}$. The laser dye is the Rhodamine 6G with a concentration of $1.8 \times 10^{-3} \mathrm{~mol} / \mathrm{l}$, and SA is DODCI with a concentration of $1.1 \times 10^{-4} \mathrm{~mol} / \mathrm{l}$. For the $3.1-\mathrm{kW}$ pumping, $q_{0} / N$ is calculated to be $56.7 \%$ from (6) and (7). Other parameters are as follows: $\sigma=1.5 \times 10^{-16} \mathrm{~cm}^{2}, \sigma_{a}=$ $1.7 \times 10^{-15} \mathrm{~cm}^{2}, \alpha=2 \mathrm{~cm}^{-1}, \alpha_{a}=2 \mathrm{~cm}^{-1}, \tau=3.5 \mathrm{~ns}$, and $\tau_{a}=0.3 \mathrm{~ns}$.

Normalized pulse shapes in each stage up to $X=4$ are shown in Fig. 5 for an input pulse energy of $0.1 \mu \mathrm{J}$. The 


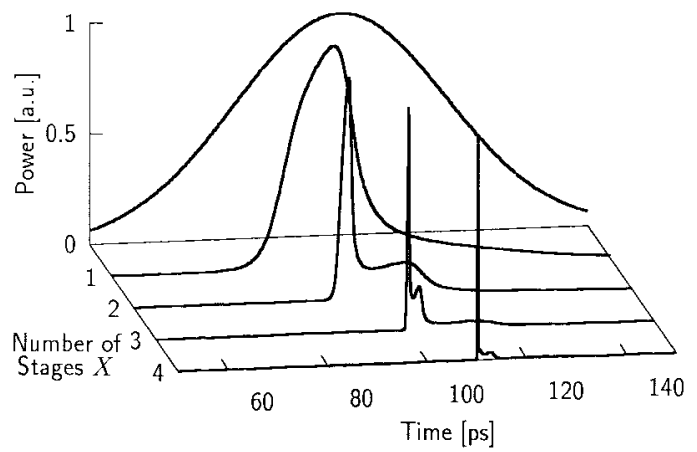

Fig. 5. An example of calculated pulse shape at each stage for 50-ps FWHM Gaussian pulse input.

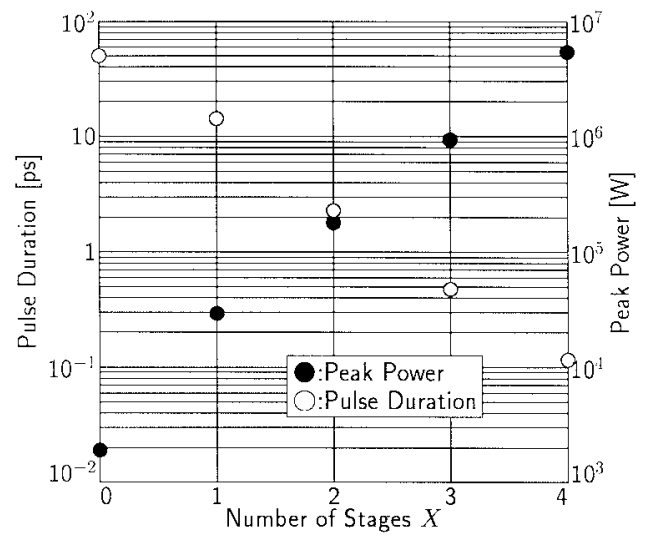

Fig. 6. Duration and peak power of pulse at each stage in the numerical calculation in Fig. 5.

pulse duration and the peak power in this case are plotted in Fig. 6. The pulse duration is compressed from 50 ps to $120 \mathrm{fs}$ for $X=4$. Since the analysis by the photon transport equation has a possibility to include some error in these very short pulse regions, this calculation is quantitatively a rough estimate. However, we think that the potentiality of this device in the efficient pulse compression was proved.

\section{CONCLUSION}

We generated a single 50-ps pulse by the pumping of a 6 -ns pulse in a thin-film DFB dye laser and proposed an integrated pulse compressor to compress it to a fs pulse. Using this technique, we expect to construct a very compact and simple ultrashort pulse generator in which whole system is integrated on a chip of a plastic waveguide.

In order to demonstrate this scheme, we are making a device with an amplifier-SA chain. In the case of a plastic dye laser, the life of the device is limited by the dye degradation, which is typically $10^{4}$ to $10^{5}$ shots. However, more than $10^{6}$ shots is possible by shifting the position of pumping. Since the pump power energy is only a few millijoules and a compact all-solid- state Nd:YAG laser can be used, we can expect a compact and low-cost ultrashort pulse generator in this scheme.

\section{REFERENCES}

[1] Zs. Bor, A. Muller, B. Racz, and F. P. Schafer, "Ultrashort pulse generation by distributed feedback dye lasers. I," Appl. Phys. B, vol. 27, pp. 9-14, 1982.

[2] _ , "Ultrashort pulse generation by distributed feedback dye lasers. II," Appl. Phys. B, vol. 27, pp. 77-81, 1982.

[3] Zs. Bor and A. Muller, "Picosecond distributed feedback dye lasers," IEEE J. Quantum Electron., vol. QE-22, pp. 1524-1533, 1986.

[4] G. Veith and A. J. Schmidt, "Generation of tunable subnanosecond laser pulses with a nitrogen laser pumped dye laser amplifier system," Opt. Commun., vol. 30, pp. 437-439, 1979.

[5] Zs. Bor, S. Szatmari, and A. Muller, "Picosecond pulse shortening by traveling wave amplified spontaneous emission," Appl. Phys. B, vol. 32, pp. 101-104, 1983.

[6] Zs. Bor and F. P. Schafer, "New single-pulse generation technique for distributed feedback dye lasers," Appl. Phys. B, vol. 1, pp. 209-213, 1983.

[7] G. Szabo, B. Racz, A. Muller, B. Nikolaus, and Zs. Bor, "Travelingwave-pumped ultrashort-pulse distributed-feedback dye laser," Appl. Phys. B, vol. 34, pp. 145-147, 1984.

[8] S. Szatmari and F. P. Schafer, "Excimer-laser-pumped ps-dye laser," Appl. Phys. B, vol. 33, pp. 95-98, 1984.

[9] Zs. Bor and B. Racz, "Picosecond dye laser pumped by an excimer laser," Appl. Opt., vol. 24, pp. 1910-1913, 1985.

[10] S. Szatmari and B. Racz, "Novel achromatic distributed feedback dye laser for subpicosecond operation," Appl. Phys. B, vol. 43, pp. 173-177, 1987.

[11] S. Szatmari and F. P. Schafer, "Subpicosecond, widely tunable distributed feedback dye laser," Appl. Phys. B, vol. 46, pp. 305-311, 1988.

[12] P. Simon, H. Gerhardt, and S. Szatmari, "Simple method for temporal study of subpicoseond distributed feedback dye lasers," Opt. Commum. vol. 71, pp. 305-309, 1989.

[13] H. Kogelnik and C. V. Shank, "Stimulated emission in a periodic structure," Appl. Phys. Lett., vol. 18, pp. 152-154, 1971.

[14] C. V. Shank, J. E. Bjorkholm, and H. Kogelnik, "Tunable distributedfeedback dye laser," Appl. Phys. Lett., vol. 18, pp. 395-396, 1971.

[15] Y. Taira and Y. Yajima, "High power subpicosecond pulse generation from a synchronously pumped dye laser combined with an external compressor," Opt. Commun., vol. 29, pp. 115-118, 1979.

[16] S. Szatmari and B. Racz, "3-ps pulse generation by excimer-laserpumped dye-laser system using gated absorbers," Opt. Quantum Electron., vol. 18, pp. 20-24, 1986.

[17] S. L. Shapiro, Ed., Ultrashort Light Pulses. Berlin, Germany: SpringerVerlag, 1976, p. 62.

[18] A. Takahashi, M. Maeda, and Y. Noda, "Short pulse generation and compression in XeCl lasers," IEEE J. Quantum Electron., vol. QE-20, pp. 1196-1201, 1984.

Mitsuo Maeda, photograph and biography not available at the time of publication.

Yuji Oki, photograph and biography not available at the time of publication.

Katsumi Imamura, photograph and biography not available at the time of publication. 